\title{
Postpartum Obstetric Air Embolism
}

National Cancer Institute

\section{Source}

National Cancer Institute. Postpartum Obstetric Air Embolism. NCI Thesaurus. Code C111781.

Presence of a large air bubble in the maternal vascular system which orig inated from a distant site during the postpartum period. 